\title{
Nature of the quantum metal in a two-dimensional crystalline superconductor
}

\author{
A. W. Tsen ${ }^{1}$, B. Hunt ${ }^{1 \dagger}$, Y. D. Kim², Z. J. Yuan ${ }^{3}$, S. Jia ${ }^{3,4}$, R. J. Cava ${ }^{5}$, J. Hone ${ }^{2}$, P. Kim ${ }^{6}$, C. R. Dean ${ }^{1 \star}$ \\ and A. N. Pasupathy ${ }^{1 \star}$
}

Two-dimensional (2D) materials are not expected to be metals at low temperature owing to electron localization ${ }^{1}$. Consistent with this, pioneering studies on thin films reported only superconducting and insulating ground states, with a direct transition between the two as a function of disorder or magnetic field ${ }^{2-6}$. However, more recent works have revealed the presence of an intermediate quantum metallic state occupying a substantial region of the phase diagram ${ }^{7-10}$, whose nature is intensely debated ${ }^{11-17}$. Here, we observe such a state in the disorder-free limit of a crystalline 2D superconductor, produced by mechanical co-lamination of $\mathrm{NbSe}_{2}$ in an inert atmosphere. Under a small perpendicular magnetic field, we induce a transition from superconductor to the quantum metal. We find a unique power-law scaling with field in this phase, which is consistent with the Bose-metal model where metallic behaviour arises from strong phase fluctuations caused by the magnetic field ${ }^{11-14}$.

Global superconductivity emerges in a sample when conduction electrons form Cooper pairs and condense into a macroscopic, phase-coherent quantum state. In two dimensions, the phase coherence can be disrupted even at zero temperature by increasing disorder-either by degrading sample quality or by applying magnetic fields to create vortices ${ }^{2}$. Granular or amorphous superconducting thin films, for which disorder levels can be varied during growth, have thus provided an established platform for the study of quantum phase transitions in 2D superconductors. Within the conventional theoretical framework, increasing sample disorder or magnetic field perpendicular to a strongly disordered film at $T=0$ induces a direct transition to an insulating state as the normal state sheet resistance approaches the pair quantum resistance $h /(2 e)^{2}=6.4 \mathrm{k} \Omega$ (refs 2,4). As film quality improved over time, however, an intervening metallic phase with resistance much lower than the normal state resistance has been observed in several systems with generally less disorder ${ }^{7-10}$. Its origin is not well understood, and the various theoretical treatments can be divided between bosonic-based models, in which Cooper pairing persists in the metallic phase but phase coherence is lost ${ }^{11-14}$, and models that also incorporate other fermionic degrees of freedom ${ }^{15-17}$.

Recently, mechanical exfoliation has emerged as a technique to produce ultraclean, crystalline $2 \mathrm{D}$ materials, with graphene being a well-known example ${ }^{18}$. Like amorphous films, the thickness of these samples can be easily controlled down to the level of individual atomic layers. In contrast to amorphous films, a 2D superconductor exfoliated from a layered, single crystal, can exist in the regime of minimal disorder, allowing new insight into the nature of the vortex state in two dimensions. In this work, we realize such a system using a clean bilayer of $\mathrm{NbSe}_{2}$, a wellknown type-II superconductor with $T_{\mathrm{c}} \sim 7 \mathrm{~K}$ in bulk form ${ }^{19,20}$. Unique to this sample, the normal state sheet resistance is two orders of magnitude below $h /(2 e)^{2}$ and insulating behaviour is never observed. Instead, the intermediate metallic phase emerges in an exceptionally large region of the magnetic field-temperature phase diagram. Unlike the typical exponential behaviour associated with the quantum tunnelling of fermionic quasiparticles ${ }^{7,15}$, we observe a new power-law scaling as a function of field at low temperature that is consistent with the Bose-metal scenario of the metallic phase $\mathrm{e}^{11-14}$

Earlier studies on isolated $\mathrm{NbSe}_{2}$ flakes do not observe a superconducting transition to a zero-resistance state in the atomically thin limit ${ }^{21,22}$. Recently, however, it has been shown the surfaces of metallic materials may oxidize, altering the electronic properties of thin samples ${ }^{23}$. Exfoliation and encapsulation by a protective layer in an inert atmosphere is thus crucial for preserving the intrinsic properties of the $2 \mathrm{D}$ material ${ }^{23,24}$. We achieve this using a mechanical transfer set-up installed inside a nitrogenfilled glove box (see Methods). In short, within the glove box, an exfoliated $\mathrm{NbSe}_{2}$ flake is electrically contacted by graphite (G), and the entire device is protected by an insulating layer of hexagonal boron nitride $(\mathrm{BN})$. The graphite leads are then contacted using an edge-metallization technique in the ambient environment ${ }^{25,26}$. A schematic depicting the assembly and fabrication process is shown in Fig. 1a and optical images of the heterostructure are shown in Fig. $1 \mathrm{~b}$ before (left) and after (right) device fabrication.

In the main panel of Fig. 2a, we show four-terminal sheet resistance as a function of temperature for a particular $\mathrm{NbSe}_{2}$ device prepared using the method described above. The $\mathrm{NbSe}_{2}$ thickness is $1.5 \mathrm{~nm}$, as determined by an atomic force microscope (AFM) outside the glovebox after BN/graphite transfer, suggesting it consists of only two atomic layers. The resistance in the normal state is $R_{\mathrm{N}}=75 \Omega$. This corresponds to a residual resistivity that is ten times larger than that of bulk crystals ${ }^{20}$, yet the sheet resistance is still an order of magnitude less than that of the most conductive amorphous superconducting films ${ }^{10}$. We observe a clear superconducting transition to a zero-resistance state measured to the limit of our instrument resolution. The critical temperature is $T_{\mathrm{c}}=5.26 \mathrm{~K}$, as defined by where the resistance is $90 \%$ of the normal

\footnotetext{
${ }^{1}$ Department of Physics, Columbia University, New York, New York 10027, USA. ${ }^{2}$ Department of Mechanical Engineering, Columbia University, New York, New York 10027, USA. ${ }^{3}$ International Center for Quantum Materials, Peking University, Beijing 100871, China. ${ }^{4}$ Collaborative Innovation Center of Quantum Matter, Beijing 100871, China. ${ }^{5}$ Department of Chemistry, Princeton University, Princeton, New Jersey 08544, USA. ${ }^{6}$ Department of Physics, Harvard University, Cambridge, Massachusetts 02138, USA. †Present address: Department of Physics, Carnegie Mellon University, Pittsburgh, Pennsylvania 15213, USA. *e-mail: cdean@phys.columbia.edu; pasupathy@phys.columbia.edu
} 
a

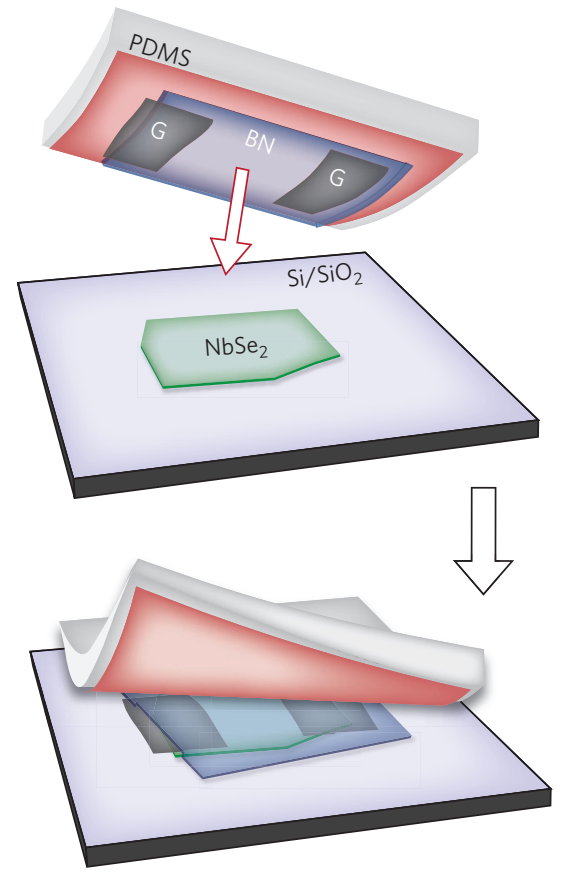

b

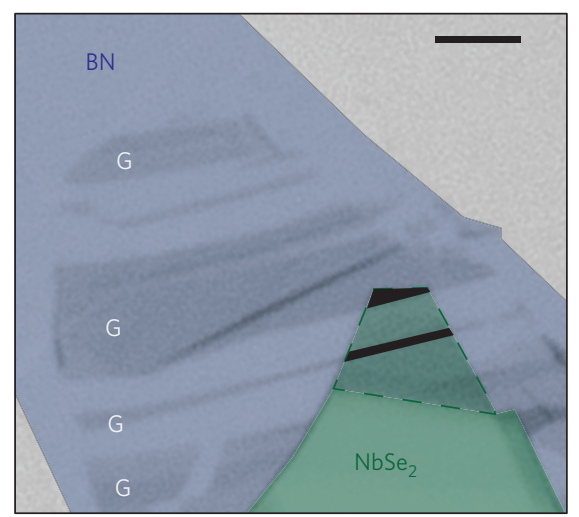

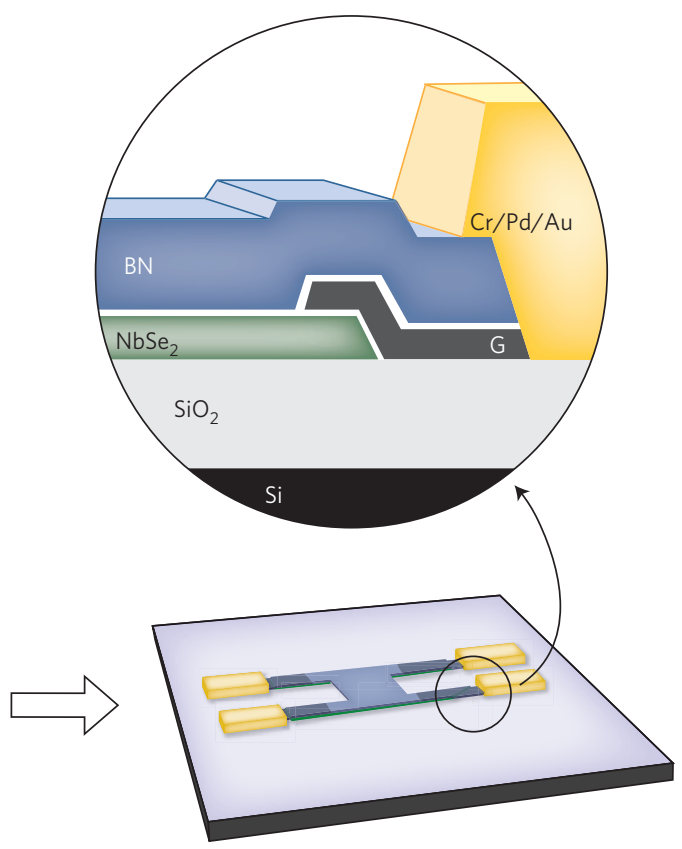

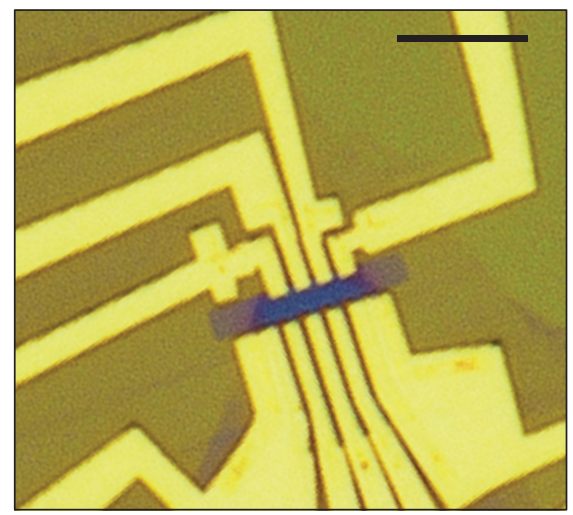

Figure 1 | Environmentally controlled device fabrication. a, Schematic of the heterostructure assembly process. Boron nitride (BN)/graphite (G) on a

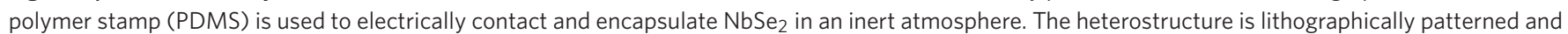
the edge of graphite is metallized with $\mathrm{Cr} / \mathrm{Pd} / \mathrm{Au}$. b, Optical images of the heterostructure before (left) and after (right) device fabrication. In the (false-coloured) left panel, the bilayer $\mathrm{NbSe}_{2}$ is outlined with a dashed green line and the overlap between the graphite and bilayer NbSe 2 is shaded black. Scale bar is $5 \mu \mathrm{m}$ in both images.

state value, which is slightly reduced from the transition temperature of bulk samples (6.8-7.2 K; refs 19,20).

To further characterize the quality and dimensionality of our sample, we measured the temperature-dependent critical fields, defined by $R\left(H_{\mathrm{c} 2}, T\right)=0.9 R_{\mathrm{N}}$, for field configurations both perpendicular and parallel to the layers, and the results are plotted in the inset of Fig. 2a. Close to $T_{\mathrm{c}}$, we expect $H_{\mathrm{c} 2}^{\perp}=\phi_{0} /\left(2 \pi \xi(0)^{2}\right)\left(1-\left(T / T_{\mathrm{c}}\right)\right)$, where $\phi_{0}$ is the flux quantum and $\xi(0)$ is the in-plane Ginzburg-Landau coherence length at zero temperature ${ }^{27}$. A linear fit shown by the black line yields $\xi(0)=8.9 \mathrm{~nm}$, similar to the bulk value ${ }^{20}$. From the normal state residual resistance and carrier concentration, as determined by Hall measurements, we estimate the electron mean free path to be $l=17 \mathrm{~nm}$, smaller than that in bulk crystals $^{20}$, but nearly twice $\xi(0)$, indicating that our device is a 'clean' superconductor. In this regime, the Bardeen-CooperSchrieffer (BCS) coherence length $\xi_{0}=1.35 \xi(0)<l$ (ref. 27). In contrast, amorphous films are generally characterized as 'dirty' superconductors with $\xi_{0} \gg l$. The critical parallel field does not exhibit the linear temperature dependence expected for anisotropic
$3 \mathrm{D}$ superconductors $\mathrm{s}^{27}$. Instead, a $2 \mathrm{D}$ superconductor with thickness $d<\xi_{0}$ obeys $H_{\mathrm{c} 2}^{\|}=\sqrt{12} \phi_{0} /(2 \pi \xi(0) d) \sqrt{1-\left(T / T_{\mathrm{c}}\right)}$ (ref. 28). The red curve in the inset of Fig. 2a shows a best fit to this expression, from which we extract $d=3.4 \mathrm{~nm}$, which is larger than the thickness as determined by AFM $(1.5 \mathrm{~nm})$. However, this fitting has been previously found to overestimate the true sample thickness in sufficiently thin systems ${ }^{28}$.

For a $2 \mathrm{D}$ system with $d<\xi(0)$, when the in-plane magnetic penetration length $\lambda^{2} / d$ exceeds the lateral sample size (assuming $\lambda=250 \mathrm{~nm}$ as in the bulk $\left.{ }^{19}: \lambda^{2} / d \sim 40 \mu \mathrm{m}\right)$, the superconducting phase transition is expected to be of the Berezinskii-KosterlitzThouless (BKT) type ${ }^{29}$. In this scenario, the low-temperature, zeroresistance phase consists of bound vortex-antivortex pairs created by thermal fluctuations. On heating, the pairs dissociate and may move, inducing dissipation. The BKT temperature defines the vortex unbinding transition and can be determined using current-voltage measurements as a function of temperature $T$, as shown in the main panel of Fig. 2b. Current excites free-moving vortices, causing a nonlinear voltage dependence: $V \sim I^{a(T)}$. At $T_{\mathrm{BKT}}$, a $2 \mathrm{D}$ superconductor obeys the universal scaling relation, 

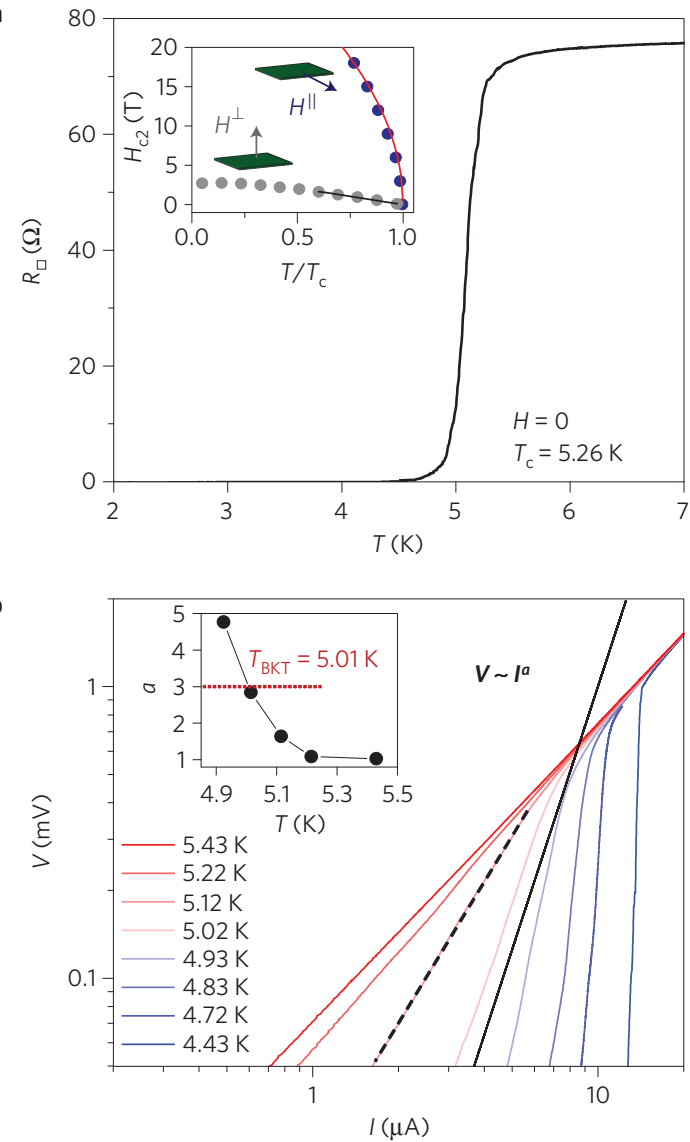

Figure $\mathbf{2}$ | Characterization of bilayer $\mathrm{NbSe}_{2}$ device. a, Sheet resistance as a function of temperature, showing a superconducting transition at $T_{\mathrm{c}}=5.26 \mathrm{~K}$. Temperature-dependent critical magnetic fields parallel and perpendicular to the layers are shown in the inset. The black line is a linear fit to $H_{\mathrm{c} 2}^{\perp} \propto 1-T / T_{\mathrm{c}}$ at high temperatures. The red line is a fit to $H_{\mathrm{c} 2}^{\|} \propto \sqrt{1-\left(T / T_{\mathrm{c}}\right)}$, the Tinkham formula for $2 \mathrm{D}$ samples $^{28}$.

b, Voltage-current behaviour at different temperatures. Inset shows the exponent $a$ versus $T$ extracted from power-law fitting $V \sim l^{a}$ near the normal state transition. $a=3$ at the BKT temperature $5.01 \mathrm{~K}$.

$V \sim I^{3}$ (solid line in Fig. $\left.2 \mathrm{~b}\right)^{29,30}$. In the inset, we plot $a$ versus $T$, as determined by the slope of the different $V-I$ traces on a $\log -\log$ scale. An example guide-to-eye fit is marked by the dashed line in the main panel. We determine $T_{\mathrm{BKT}}=5.01 \mathrm{~K}$ from where $a=3$ interpolates, only slightly less than $T_{\mathrm{c}}$ as defined above. This is consistent with the behaviour of systems with normal state resistance much less than $h /(2 e)^{2}$, where $T_{\mathrm{BKT}}$ is expected to be very close to the mean-field transition temperature ${ }^{31}$.

The measurements performed above confirm that our device exhibits the characteristics of a true $2 \mathrm{D}$ superconductor. The extracted material parameters together with the low normal state sheet resistance further places the high sample quality within a previously unexplored regime. We next turn to the dependence of resistance on perpendicular magnetic field, as the effect of vortices can now be cleanly separated from the low static disorder present in the sample. Shown in Fig. $3 \mathrm{a}$ is a $2 \mathrm{D}$ colour map of the fourterminal sheet resistance in the same device as a function of both temperature and magnetic field applied perpendicular to the layers. For clarity, temperature traces for different field levels are shown in an Arrhenius plot in Fig. 3b. For fields larger than $H_{c 2} \sim 3 \mathrm{~T}$, the sample is in the normal state for all temperatures. Previous works on strongly disordered films observed a field-tuned transition to an insulating state of bosons ${ }^{5,6}$; however, one might not expect insulating behaviour for finite samples of a highly conductive 2D system in the disorder-free limit ${ }^{10}$. We have applied perpendicular fields as large as $14.5 \mathrm{~T}$ and still see metallic behaviour in the temperature dependence (see Supplementary Fig. 1 main panel).

As one lowers the field to just below $H_{\mathrm{c} 2}$, a resistance drop is observed on cooling from the normal state. In this region of the $H-T$ phase diagram, the device exhibits activated behaviour, as can been seen in the linear slope in the Arrhenius plot (black lines in Fig. 3b). Classically, dissipation in a superconductor in which resistance is less than the normal state value can be attributed to the motion of individual vortices (flux creep or flow) ${ }^{27}$. In a clean 2D system, we expect the dominant energy barrier to flux motion to be that of vortex-antivortex dissociation ${ }^{32}$. Flux resistance then becomes thermally activated when the temperature is comparable to the barrier energy. We have determined the activation energy from the linear portion of the Arrhenius plot in Fig. 3b for different magnetic fields, and the result is plotted in Fig. 3c. The functional form is expected to be $U(H)=U_{0} \ln \left(H_{0} / H\right)$, where $U_{0} \sim\left(\phi_{0}{ }^{2} d\right) /\left(256 \pi^{3} \lambda^{2}\right)$, the vortex-antivortex binding energy, and $H_{0} \sim H_{\mathrm{c} 2}$ (refs 7,32). A fit to this form yields $U_{0}=27.5 \mathrm{~K}$ and $H_{0}=2 \mathrm{~T}$ for our device. For $\lambda=250 \mathrm{~nm}$ (ref. 19), we estimate $U_{0} \sim 10 \mathrm{~K}$, of the order of the experimental value.

At lower temperatures, the resistance saturates to a level dependent on magnetic field (see coloured lines in Fig. 3b), hallmark of the quantum metallic state that is the main subject of this report. Qualitatively similar behaviour has also been observed in
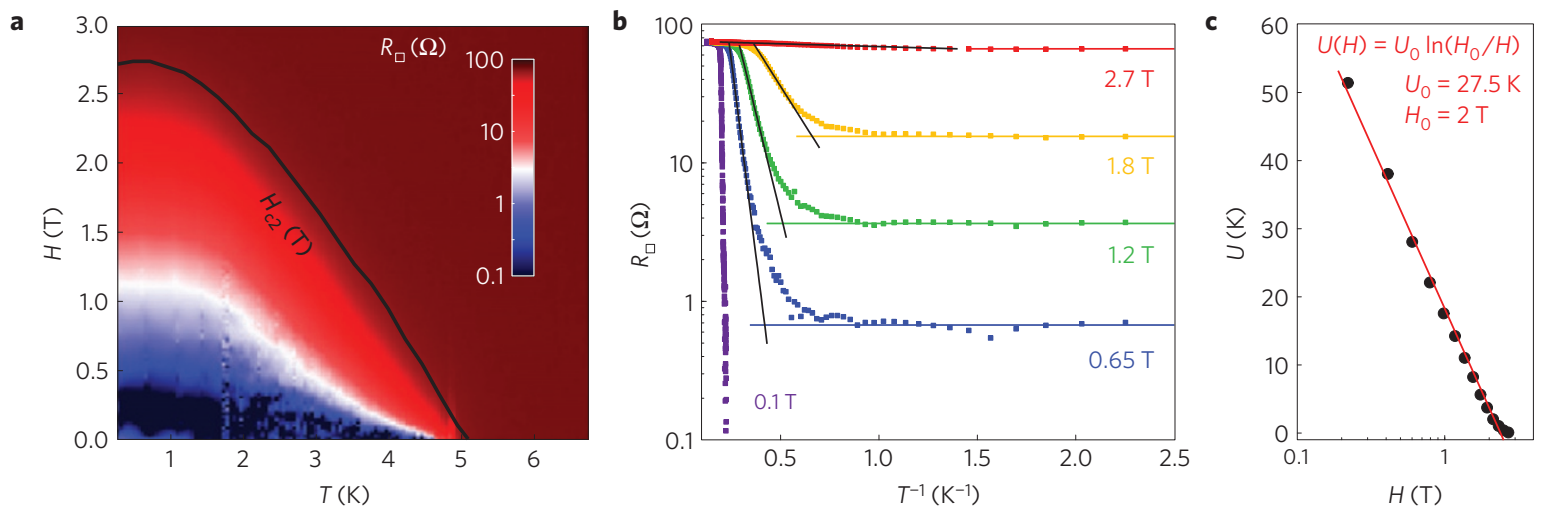

Figure 3 | Magnetic-field-tuned phase transitions in 2D NbSe2. $\mathbf{a}, 2 \mathrm{D}$ colour plot of sheet resistance versus temperature and perpendicular magnetic field. The black line marks $H_{\mathrm{c} 2}(T)$. b. Arrhenius plot of resistance for several magnetic fields shows the thermally activated regime (black lines) and saturation at low temperatures (coloured lines). c, Energy barrier versus magnetic field extracted from a linear fit to the activated region. The solid red line is an empirical fit to the formula given as an inset. 
amorphous MoGe and Ta films with much larger normal state sheet resistances ${ }^{7,9}$. This effect cannot be understood within a classical framework, in which we expect to recover superconductivity for $T \ll U$. In the past, various theories have been advanced to explain the origin of dissipation in this temperature regime for disordered films ${ }^{11-17}$. One can distinguish between the different models on the basis of the magnetic field dependence of the saturated resistance at low temperatures.

Shimshoni et al. consider a disordered superconducting film as a percolating network of superconducting islands within an insulating matrix ${ }^{15}$. Field-induced vortices that tunnel across thin superconducting constrictions give rise to resistance when coupled to a fermionic bath. The resistance depends on field as

$$
R=\frac{h}{4 e^{2}} \exp \left[C \frac{\pi}{2}\left(\frac{\hbar / e^{2}}{R_{\mathrm{N}}}\right)\left(\frac{H-H_{\mathrm{c} 2}}{H_{\mathrm{c} 2}}\right)\right]
$$

where $R_{\mathrm{N}}$ is the normal state resistance and $C$ is a dimensionless constant of order unity. This expression finds good agreement with the measurements of Ephron et al. on amorphous MoGe films, in which $R_{\mathrm{N}} \sim 1 \mathrm{k} \Omega$ (ref. 7), but evidently provides a poor fit to our data (see Supplementary Fig. 2). Furthermore, because our device is over an order of magnitude more conductive, the closest fitting requires $C=0.14$, an unphysically small value.

Galitski et al. consider a 'vortex metal' phase where field-induced vortices interact with electrically neutral spinons ${ }^{17}$. The theory accounts for the large peak in low-temperature magnetoresistance observed in disordered InOx films before saturation to $R_{\mathrm{N}}$ at higher fields ${ }^{10}$. In our device, however, we observe a monotonic dependence of resistance on field and see no peak structure in magnetoresistance up to 7 T (see Supplementary Fig. 1 inset).

Das and Doniach, as well as Dalidovich and Phillips, report that a 2D system of interacting bosons may form a gapless, nonsuperfluid state in the limit of zero temperature, a phase which they term a 'Bose metal' ${ }^{11-14}$. They argue that the uncondensed Cooper pairs and vortices are responsible for the small resistance observed at small finite field ${ }^{12}$. A magnetic field introduces gauge fluctuations, which disrupt phase coherence and cause dissipation, a quantum analogue of that caused by thermal fluctuations in the BKT transition. In the Bose-metal model, resistance on the metallic side of the field-tuned transition can be described by: $R \sim\left(H-H_{\mathrm{c} 0}\right)^{2 v}$, where $H_{\mathrm{c} 0}$ is the critical field of the superconductor to Bose-metal transition and $v$ is the exponent of the superfluid correlation length, which diverges across the boundary as $\left(H-H_{\mathrm{c} 0}\right)^{-v}$ (ref. 12).

In the main panel of Fig. $4 \mathrm{a}$, we show a log-log plot of $R$ versus $\left(H-H_{\mathrm{c} 0}\right)$ taken at several different temperatures. $H_{\mathrm{c} 0}$ is a small temperature-dependent value which we determined using a method that shall be described below. The linear scaling observed here suggests a power-law dependence on field. We have fitted the data to the expression $R \sim\left(H-H_{\mathrm{c} 0}\right)^{\alpha}$ and the extracted exponent $\alpha$ is plotted in the inset as a function of temperature. At high temperatures, but below $T_{c}$, resistance increases roughly linearly with field, as expected for unhindered flux flow: $R \sim R_{\mathrm{N}} H / H_{\mathrm{c} 2}(\alpha=1)$ (ref. 27). The grey line shows linear scaling as a guide to eye. As temperature is lowered, the field dependence becomes increasingly nonlinear and collapses onto a single curve below $1 \mathrm{~K}$ with $\alpha \sim 3$. The red line is an empirical fit given by $R[\Omega]=5.44(H[\mathrm{~T}]-0.15)^{3.21}$, which shows excellent agreement with the data. This yields a critical exponent of $v=1.61$. Previous measurements on MoGe films at the lowest accessible temperatures observe mostly an exponential dependence of resistance with field ${ }^{7,33}$, which is consistent with quantum tunnelling of fermionized vortices ${ }^{15}$. At small very fields for the MoGe film, however, the scaling obeys a power law with unity exponent $^{33}$. The power-law scaling we observe in our sample consistently over the entire field range thus suggests that fermionic

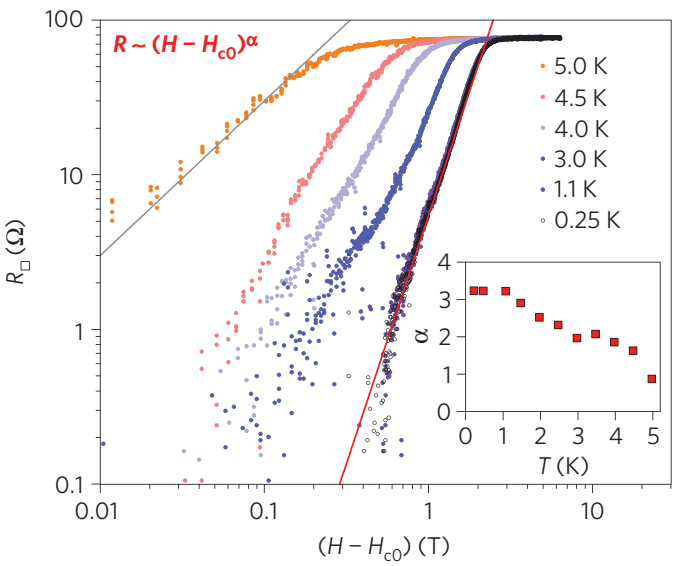

b

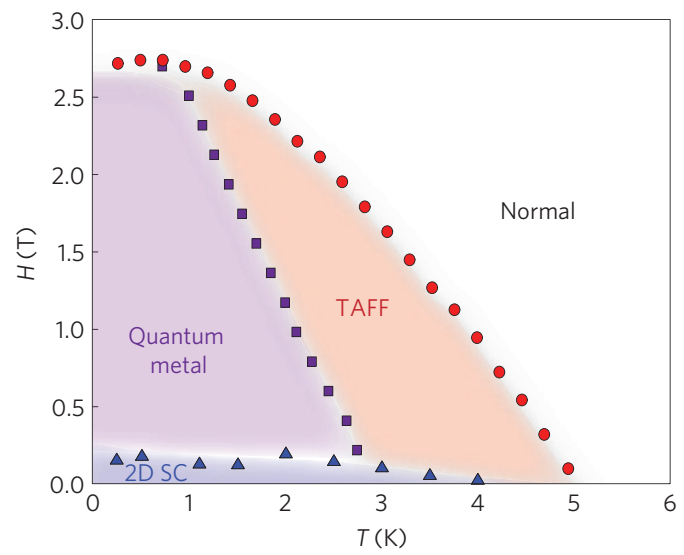

Figure 4 | Emergence of the quantum metal. a, Magnetoresistance below the superconducting transition for different temperatures. The data scale to a power law $R \sim\left(H-H_{\mathrm{CO}}\right)^{\alpha(T)}$ and collapse onto a single curve in the quantum metallic phase below $1 \mathrm{~K} . \alpha$ versus $T$ is plotted in the inset. The grey line is a guide-to-eye linear scaling (high $T$ ) and the red line is an empirical fit to Bose-metal scaling (low $T$ ). b. Full $\mathrm{H}$ - $\mathrm{T}$ phase diagram of the bilayer $\mathrm{NbSe}_{2}$ device. The red circles are $H_{\mathrm{c} 2}(T)$. The purple squares, dividing the Bose metal from the thermally assisted flux flow (TAFF) regime, mark the transition from activated behaviour $R \sim \exp (U(H) / T)$ to temperature-independent resistance $R=R(H)$, that is, the intersection of the black and coloured lines in Fig. 3b. The blue triangles denote the boundary of the superconducting phase $H_{c 0}(T)$. This criterion is determined by when hysteresis vanishes in $V$-I measurements (see Supplementary Fig. 4).

tunnelling undergoes a crossover to Bose-metal behaviour in the limit of vanishing static disorder. We also found similar field scaling in the quantum metallic state for other ultrathin devices, whereas this phase is distinctly absent in the bulk crystal (see Supplementary Fig. 3).

The critical field of the transition out of the true zero-resistance state $H_{\mathrm{c} 0}$ is difficult to determine directly from the linear resistance, given the limited accuracy of our instruments. Recently, however, experiments by Qin et al. and $\mathrm{Li}$ et al. on disordered Ta films showed that $H_{\mathrm{c} 0}$ can be determined indirectly using current-voltage measurements $s^{9,34}$. Hysteresis is observed in the $V-I$ characteristics on the superconducting side, similar to that seen in underdamped Josephson junctions ${ }^{27}$, which disappears at the onset of the metallic phase. We have performed $V-I$ measurements on the same bilayer device at $T=0.5 \mathrm{~K}$ for increasing magnetic fields (see Supplementary Fig. 4 main panel). Clear hysteresis is seen between current sweeps up and down for low fields. In the inset, we plot the current hysteresis $\Delta I$ at which the voltage jump occurs as a function of magnetic field. $\Delta I$ vanishes close to $H_{\mathrm{c} 0}=0.175 \mathrm{~T}$. 
This then allows us to identify the true superconducting phase as that for $H<H_{\mathrm{c} 0}$. We have repeated this measurement for several different temperatures to determine the critical field as a function of temperature.

Figure $4 \mathrm{~b}$ shows a full $H-T$ phase diagram for our device. The blue triangles mark $H_{c 0}(T)$ and the red circles mark $H_{c 2}^{\perp}(T)$. The boundary between Bose metal and flux flow (purple squares) is defined by the intersection of the fits to the activated resistance and saturated levels in Fig. 3b. The normal phase extends up to at least 14.5 $\mathrm{T}$ without the appearance of an insulating phase, in contrast to previous works on strongly disordered films. The high sample quality is made possible by our facile device assembly technique in an inert atmosphere, which demonstrates a new route for the production of 2D superconductors in the ultraclean limit.

\section{Methods}

Methods and any associated references are available in the online version of the paper.

Received 21 August 2015; accepted 25 October 2015; published online 7 December 2015

\section{References}

1. Abrahams, E., Anderson, P. W., Licciardello, D. C. \& Ramakrishnan, T. V. Scaling theory of localization: Absence of quantum diffusion in two dimensions. Phys. Rev. Lett. 42, 673-676 (1979).

2. Goldman, A. M. \& Markovic, N. Superconductor-insulator transitions in the two-dimensional limit. Phys. Today 51, 39-44 (1998).

3. Haviland, D. B., Liu, Y. \& Goldman, A. M. Onset of superconductivity in the two-dimensional limit. Phys. Rev. Lett. 62, 2180-2183 (1989).

4. Fisher, M. P. A. Quantum phase transitions in disordered two-dimensional superconductors. Phys. Rev. Lett. 65, 923-926 (1990).

5. Hebard, A. F. \& Paalanen, M. A. Magnetic-field-tuned superconductor-insulator transition in two-dimensional films. Phys. Rev. Lett. 65, 927-930 (1990)

6. Yazdani, A. \& Kapitulnik, A. Superconducting-insulating transition in two-dimensional $\alpha$-MoGe thin films. Phys. Rev. Lett. 74, 3037-3040 (1995).

7. Ephron, D., Yazdani, A., Kapitulnik, A. \& Beasley, M. R. Observation of quantum dissipation in the vortex state of a highly disordered superconducting thin film. Phys. Rev. Lett. 76, 1529-1532 (1996).

8. Christiansen, C., Hernandez, L. M. \& Goldman, A. M. Evidence of collective charge behavior in the insulating state of ultrathin films of superconducting metals. Phys. Rev. Lett. 88, 037004 (2002).

9. Qin, Y. G., Vicente, C. L. \& Yoon, J. Magnetically induced metallic phase in superconducting tantalum films. Phys. Rev. B 73, 100505 (2006).

10. Steiner, M. A., Breznay, N. P. \& Kapitulnik, A. Approach to a superconductor-to-Bose-insulator transition in disordered films. Phys. Rev. B 77, 212501 (2008).

11. Das, D. \& Doniach, S. Existence of a Bose metal at T=0. Phys. Rev. B 60, 1261-1275 (1999).

12. Das, D. \& Doniach, S. Bose metal: Gauge-field fluctuations and scaling for field-tuned quantum phase transitions. Phys. Rev. B 64, 134511 (2001).

13. Dalidovich, D. \& Phillips, P. Phase glass is a Bose metal: A new conducting state in two dimensions. Phys. Rev. Lett. 89, 027001 (2002).

14. Phillips, P. \& Dalidovich, D. The elusive Bose metal. Science 302, 243-247 (2003).

15. Shimshoni, E., Auerbach, A. \& Kapitulnik, A. Transport through quantum melts. Phys. Rev. Lett. 80, 3352-3355 (1998).

16. Spivak, B., Zyuzin, A. \& Hruska, M. Quantum superconductor-metal transition. Phys. Rev. B 64, 132502 (2001).

17. Galitski, V. M., Refael, G., Fisher, M. P. A. \& Senthil, T. Vortices and quasiparticles near the superconductor-insulator transition in thin films. Phys. Rev. Lett. 95, 077002 (2005)
18. Geim, A. K. \& Novoselov, K. S. The rise of graphene. Nature Mater. 6 , 183-191 (2007)

19. Le, L. P. et al. Magnetic penetration depth in layered compound $\mathrm{NbSe}_{2}$ measured by muon spin relaxation. Physica C 185, 2715-2716 (1991).

20. Soto, F. et al. Electric and magnetic characterization of $\mathrm{NbSe}_{2}$ single crystals: Anisotropic superconducting fluctuations above $T_{\mathrm{c}}$. Physica $C \mathbf{4 6 0}$, 789-790 (2007)

21. Staley, N. E. et al. Electric field effect on superconductivity in atomically thin flakes of $\mathrm{NbSe}_{2}$. Phys. Rev. B 80, 184505 (2009).

22. El-Bana, M. S. et al. Superconductivity in two-dimensional $\mathrm{NbSe}_{2}$ field effect transistors. Supercond. Sci. Technol. 26, 125020 (2013).

23. Tsen, A. W. et al. Structure and control of charge density waves in two-dimensional 1T-TaS 2 . Proc. Natl Acad. Sci. USA http://dx.doi.org/10.1073/pnas.1512092112 (in the press).

24. Cao, Y. et al. Quality heterostructures from two-dimensional crystals unstable in air by their assembly in inert atmosphere. Nano Lett. 15, 4914-4921 (2015).

25. Wang, L. et al. One-dimensional electrical contact to a two-dimensional material. Science 342, 614-617 (2013).

26. Cui, X. et al. Multi-terminal transport measurements of $\mathrm{MoS}_{2}$ using a van der Waals heterostructure device platform. Nature Nanotech. 10, 534-540 (2015).

27. Tinkham, M. Introduction to Superconductivity 2nd (Dover, 1996)

28. Kim, M., Kozuka, Y., Bell, C., Hikita, Y. \& Hwang, H. Y. Intrinsic spin-orbit coupling in superconducting $\delta$-doped $\mathrm{SrTiO}_{3}$ heterostructures. Phys. Rev. B 86, 085121 (2012)

29. Halperin, B. I. \& Nelson, D. R. Resistive transition in superconducting films. J. Low Temp. Phys. 36, 599-616 (1979)

30. Eley, S., Gopalakrishnan, S., Goldbart, P. M. \& Mason, N. Approaching zero-temperature metallic states in mesoscopic superconductor-normal-superconductor arrays. Nature Phys. 8, 59-62 (2012).

31. Beasley, M. R., Mooij, J. E. \& Orlando, T. P. Possibility of vortex-antivortex pair dissociation in two-dimensional superconductors. Phys. Rev. Lett. 42, 1165-1168 (1979).

32. Feigelman, M. V., Geshkenbein, V. B. \& Larkin, A. I. Pinning and creep in layered superconductors. Physica C 167, 177-187 (1990).

33. Mason, N. \& Kapitulnik, A. True superconductivity in a two-dimensional superconducting-insulating system. Phys. Rev. B 64, 060504 (2001).

34. Li, Y., Vicente, C. L. \& Yoon, J. Transport phase diagram for superconducting thin films of tantalum with homogeneous disorder. Phys. Rev. B 81, 020505 (2010)

\section{Acknowledgements}

We acknowledge helpful discussions with Z. Han, J.-D. Pillet, E. Shimsoni, O. Vafek, A. Kapitulnik, D. Xiao and D. Gopalan. We thank J. Shi, F. Zhao, D. Wang and S. Chen for assistance with device fabrication. This material is based on work supported by the NSF MRSEC Program through Columbia in the Center for Precision Assembly of Superstratic and Superatomic Solids (DMR-1420634). Salary support is provided by the NSF under grants NEB- 1124894 (A.W.T.) and DMR-1056527 (A.N.P.). Some measurements were performed at the National High Magnetic Field Laboratory, which is supported by the NSF Cooperative Agreement (DMR-0654118), the State of Florida and the Department of Energy. S.J. is supported by the National Basic Research Program of China (grants 2013CB921901 and 2014CB239302). R.J.C. is supported by the Department of Energy, Division of Basic Energy Sciences (grant DOE FG02-98ER45706). P.K. acknowledges support from the Army Research Office (grant W911NF-14-1-0638).

\section{Author contributions}

A.W.T., B.H., C.R.D. and A.N.P. conceived and designed the experiment; Z.J.Y. and S.J. synthesized the $\mathrm{NbSe}_{2}$ crystals; A.W.T. fabricated the devices with assistance from Y.D.K.; A.W.T. and B.H. performed the transport measurements; A.W.T., B.H., C.R.D. and A.N.P. analysed the data and wrote the paper. R.J.C., J.H., P.K., C.R.D. and A.N.P. advised.

\section{Additional information}

Supplementary information is available in the online version of the paper. Reprints and permissions information is available online at www.nature.com/reprints. Correspondence and requests for materials should be addressed to C.R.D. or A.N.P.

\section{Competing financial interests}

The authors declare no competing financial interests. 


\section{Methods}

Crystal synthesis. Polycrystalline $\mathrm{NbSe}_{2}$ was made by heating stoichiometric amounts of $\mathrm{Nb}$ powder (99.5\%) and Se shots (99.999\%) in evacuated silica ampules. Single crystals of $\mathrm{NbSe}_{2}$ were grown using a vapour transfer method. $400 \mathrm{mg}$ of $\mathrm{NbSe}_{2}$ powder and $80 \mathrm{mg}$ of $\mathrm{I}_{2}$ were sealed in a $23-\mathrm{cm}$-long silica ampule with a $1.13 \mathrm{~cm}^{2}$ inner cross section. The charge was put in the hot zone of $850^{\circ} \mathrm{C}$ and the sink in the cold zone of $750{ }^{\circ} \mathrm{C}$. After one week, all the polycrystals became single crystals, with most of the thicker plates found in the hot zone.

Device assembly and fabrication. We have exfoliated ultrathin $\mathrm{NbSe}_{2}$ flakes in a nitrogen-filled glove box containing less than 2 ppm oxygen. Separately, we prepared thin hexagonal boron nitride (BN) on a polydimethylsiloxane (PDMS) stamp covered with polypropylene carbonate (PPC), which we use to 'pick up' two closely spaced graphite flakes (separation $\sim 2 \mu \mathrm{m}$ ). The BN/graphite stack is then used to cover the $\mathrm{NbSe}_{2}$ flake inside the glove box. While graphite makes electrical contact to $\mathrm{NbSe}_{2}, \mathrm{hBN}$ provides an insulating oxidation barrier. Subsequent lithography may then be used to define a four-terminal device, in which the gapped region between the graphite leads form the channel. The graphite is then electrically contacted using an edge-metallization technique ${ }^{25,26}$. 